Objectives: The main of this study was to examine the relationship between plasma homocysteine (Hcy), asymptomatic osteoporotic vertebral fractures (VFs) using vertebral fracture assessment (VFA) and prevalent abdominal aortic calcification (AAC) in Moroccan postmenopausal women.

Methods: The study cohort consisted of 188 consecutive postmenopausal women with no prior known diagnosis of osteoporosis or taking medication interfering with bone metabolism. Mean age, weight, height, body mass index and plasma homocysteine were determined. Lateral VFA images and scans of the lumbar spine and proximal femur were obtained using a Lunar Prodigy Vision densitometer (GE Healthcare Inc., Waukesha, WI). VFs were defined using a combination of Genant's semiquantitative approach and morphometry. VFA images were also scored for prevalent AAC using a validated 24 point scale.

Results: Fifty-eight (30.9\%) patients had densitometric osteoporosis. VFs were identified using VFA in $76(40.4 \%)$ patients: 61 women had grade 1 VFs and 15 had grade 2 or 3 VFs. One hundred twenty nine women $(68.6 \%)$ did not have any detectable $\mathrm{AAC}$, whereas the prevalence of significant atherosclerotic burden defined as AAC score of 5 or higher, was $13.8 \%$. A significant positive correlation between AAC score and homocysteine was observed. Women with extended AAC, were older, had a lower weight, BMI and BMD, higher homocysteine levels and more prevalent VFs than women without extended AAC. Multiple regression analysis showed that the presence of extended AAC was significantly associated with Age and grade 2/3 VFs and not independently associated with homocysteine levels.

Conclusions: This study did not confirm that homocysteine is important determinant of extended AAC in postmenopausal women. However, this significant atherosclerotic marker is independently associated with VFs regardless of age.

Disclosure of Interest: None declared

DOI: 10.1136/annrheumdis-2017-eular.1447

\section{FRI0549 IMPACT OF CHEMOTHERAPY ON BONE MINERAL DENSITY IN POSTMENOPATHIC WOMEN WITH BREAST CANCER IN TREATMENT WITH AROMATASE INHIBITORS}

J. Loarce-Martos ${ }^{1}$, W.A. Sifuentes Giraldo ${ }^{1}$, L.V. Maldonado Romero ${ }^{1}$, M. Ahijón Lana ${ }^{1}$, C. Velázquez Arce ${ }^{1}$, N. Martínez Jañez ${ }^{2}, M^{2}$. Vázquez Díaz ${ }^{1}$. ${ }^{1}$ Rheumatology; ${ }^{2}$ Oncology, Ramon y Cajal University Hospital, Madrid, Spain

Background: Aromatase inhibitors (Al) been related to an increased risk of bone loss and fractures in women receiving these drugs as adjuvant treatment, but few studies have assessed the impact of prior chemotherapy (CT) on bone mineral density (BMD) loss associated to Al.

Objectives: To assess the impact of CT prior to the initiation of Al on BMD in postmenopausal patients with breast cancer (BC) seen at a Spanish tertiary care hospital.

Methods: We perform a longitudinal study in patients who received Al after initial CT (CT group) or as adjuvant therapy without prior CT (non-CT group) followed up for 12 months. BMD was assessed by DXA in lumbar spine (LS) and femoral neck (FN) at baseline and after 12 months of $\mathrm{Al}$ treatment following the usual protocol of our center, with in vitro coefficient of variation of $1 \%$ in both locations and estimated minimal significant change (MSC) of $0.0223 \mathrm{~g} / \mathrm{cm}^{2}$ in LS and $0.0288 \mathrm{~g} / \mathrm{cm}^{2}$ in FN. Demographics, neoplastic disease data, and osteoporosis risk factors were also collected.

Results: 69 patients (CT group 39, non-CT group 30) attended at our center between August 2011 and December 2014 were included. Mean age at diagnosis was $59.9 \pm 7.7$ years, most of them have BC stages I-II (84\%). Most frequent Al in both groups was letrozole (95\%). Baseline characteristics were similar, except for age at diagnosis that was significantly higher in the non-CT group, these data are presented in the table. Mean BMD at the start of $\mathrm{Al}$ was significantly lower in LS in the CT group $\left(0.7793 \mathrm{~g} / \mathrm{cm}^{2}\right)$ than in the non-CT group $\left(0.8483 \mathrm{~g} / \mathrm{cm}^{2}\right)$ $(p=0.018)$, but no difference in FN (CT $0.6764 \mathrm{~g} / \mathrm{cm}^{2}$ and non-CT $0.7077 \mathrm{~g} / \mathrm{cm}^{2}$, $\mathrm{p}=0.123$ ). A significant difference in LS (CT $0.7685 \mathrm{~g} / \mathrm{cm}^{2}$, non-CT $0.8397 \mathrm{~g} / \mathrm{cm}^{2}$, $p=0.003$ ) was found in the comparison of BMD means between the two groups at 12 months but not in FN (CT $0.6598 \mathrm{~g} / \mathrm{cm}^{2}$, non-CT $0.6689 \mathrm{~g} / \mathrm{cm}^{2}, \mathrm{p}=0.369$ ). After 12 months of treatment with $\mathrm{Al}$, mean $\mathrm{BMD}$ change in the CT group in LS was $-0.0107 \mathrm{~g} / \mathrm{cm}^{2}(95 \%$ confidence interval $[\mathrm{Cl}]-0.0269,+0.0055, \mathrm{p}=0.189)$ and in FN $-0.0165 \mathrm{~g} / \mathrm{cm}^{2}(95 \% \mathrm{Cl}:-0.0339,+0.0009, \mathrm{p}=0.063)$, while in the non-CT group the means changes were in LS $-0.0085 \mathrm{~g} / \mathrm{cm}^{2}(95 \% \mathrm{Cl}-0.0416,+0.0244$, $\mathrm{p}=0.599)$ and $\mathrm{FN}-0.0388 \mathrm{~g} / \mathrm{cm}^{2}(95 \% \mathrm{Cl}-0.0707,-0.0068, \mathrm{p}=0.019)$. During the study period there was a fracture in each group (CT $2.6 \%$, non-CT $3.3 \%$ ).

\begin{tabular}{lccc}
\hline & CT group $(\mathrm{n}=39)$ & Non-CT group $(\mathrm{n}=30)$ & $\mathrm{p}$-value \\
\hline Age at diagnosis, years (mean $\pm \mathrm{SD})$ & $58.2 \pm 7.3$ & $62 \pm 7.9$ & $0.042^{*}$ \\
Age of menarche, years (mean \pm SD) & $12.5 \pm 1.2$ & $12.5 \pm 1.1$ & 0.980 \\
Age of menopause, years (mean \pm SD) & $48 \pm 4.2$ & $48.9 \pm 4.1$ & 0.394 \\
Age $>65$ years & $13(33 \%)$ & $9(30 \%)$ & 0.284 \\
Body mass index (mean \pm SD) & $25.8 \pm 6$ & $27.6 \pm 4.3$ & 0.184 \\
Osteopenia/osteoporosis before Al & $35(89 \%)$ & $26(86 \%)$ & 0.692 \\
Smoking & $5(13 \%)$ & $2(6 \%)$ & 0.401 \\
Previous fracture & $2(5 \%)$ & $2(7 \%)$ & 0.786 \\
Family history of fracture & $2(5 \%)$ & $2(7 \%)$ & 0.786 \\
Calcium + Vitamin D Supplements & $26(67 \%)$ & $14(47 \%)$ & 0.095 \\
Radiotherapy & $27(69 \%)$ & $23(77 \%)$ & 0.493 \\
Prior tamoxifen & $5(13 \%)$ & $3(10 \%)$ & 0.717 \\
Bisphosphonates & $2(5 \%)$ & $1(3 \%)$ & 0,717 \\
\hline
\end{tabular}

Conclusions: Our results do not demonstrate that CT prior to Al treatment significantly decreased BMD during the first year. Mean change in both LS and FN in CT group was not superior to MSC nor to the change in non-CT group, although they had a significantly lower mean BMD in LS than the latter group and this difference was maintained at the end of the study period.

Disclosure of Interest: None declared

DOI: 10.1136/annrheumdis-2017-eular.6391

\section{FRI0550 MALE PATIENTS WITH RHEUMATOID ARTHRITIS HAVE AN INCREASED RISK OF OSTEOPOROSIS: FREQUENCY AND RISK FACTORS}

J.-H. Park ${ }^{1}$, S.-G. Lee ${ }^{1}$, E.-K. Park ${ }^{1}$, S.-M. Kweon ${ }^{1}$, D.H. Sohn ${ }^{2}$, Y.-K. Kim ${ }^{3}$ H.-S. Tag ${ }^{3}$, G.-T. Kim ${ }^{3}$, J.-W. Lee ${ }^{4} .{ }^{1}$ Internal Medicine, Pusan National University School of Medicine, Pusan National University Hospital; ${ }^{2}$ Microbiology and Immunology, Pusan National University School of Medicine: ${ }^{3}$ Internal Medicine, Kosin University College of Medicine: ${ }^{4}$ Internal Medicine, Busan St. Mary's Hospital, Busan, Korea, Republic Of

Background: Osteoporosis is a well-known extra-articular manifestation of rheumatoid arthritis (RA) and almost 2 times higher prevalence of osteoporosis was reported in female patients with RA than in healthy subjects. Accordingly, patients with RA are at increased risk of fragility fractures that lead to significant morbidity and mortality and higher healthcare cost. However, most previous epidemiologic studies regarding osteoporosis in RA have focused on female subjects, and little attention has been given to male patients with RA.

Objectives: To compare the prevalence of osteoporosis between male patients with RA and healthy subjects and to identify the risk factors of osteoporosis in male patients with RA.

Methods: By using a cross-sectional design, we recruited 76 male patients with RA aged 50 years and over and 76 sex-matched and age-matched healthy subjects at a university-affiliated rheumatology centre in South Korea from August 2014 to August 2016. We measured bone mineral density (BMD) at L1-4 levels of the lumbar spine and the hip (femoral neck and total hip) in all the subjects by using dual-energy X-ray absorptiometry (DEXA). We assessed the prevalence of osteoporosis defined as a T-score of $\leq-2.5$ according to the WHO criteria. We also investigated potential risk factors of decreased BMD and the presence of osteoporosis in male patients with RA using linear and logistic regression analyses, respectively.

Results: The mean age and body mass index (BMI) of the male patients with RA were 64.5 years and $22 \mathrm{~kg} / \mathrm{m}^{2}$, respectively, which were comparable with those of the healthy controls. The overall prevalence of osteoporosis at either the spine or the hip in the male patients with RA was significantly higher than that of the healthy controls (22.4\% vs $10.5 \%$, respectively; $\mathrm{p}=0.049)$. However, no significant differences in the prevalence of osteoporosis at the spine $(19.7 \%$ vs $10.5 \%$, respectively; $p=0.113$ ) and the hip ( $3.9 \%$ vs $0 \%$, respectively; $p=0.245$ ) were found between the patients with RA and the controls. For the male patients with RA, the median disease duration was 37 months, the mean 28-joint Disease Activity Score using erythrocyte sedimentation rate (DAS28-ESR) was 3.28 and the median modified total Sharp score was 6 . An increased titre of anti-cyclic citrullinated antibody showed a trend toward lower L1-4 BMD $(\beta=-0.0007, p=0.057)$ in the multivariable linear regression analysis. In addition, DAS28-ESR of $>3.2$ was independently associated with the presence of osteoporosis (OR=3.85, 95\% $\mathrm{Cl}=1.13-13.17, \mathrm{p}=0.032$ ) after adjusting for confounding factors. The patients with RA whose BMls were $\leq 22 \mathrm{~kg} / \mathrm{m}^{2}$ had a higher risk of osteoporosis ( $\mathrm{OR}=3.43$, $95 \% \mathrm{Cl}=1.04-11.33, \mathrm{p}=0.043$ ).

Conclusions: Similar to their female counterparts, the frequency of osteoporosis in male patients with RA had an osteoporosis prevalence of about 2.1 times higher than that of the healthy subjects. Increased DAS28-ESR was an independent risk factor of osteoporosis. Our data suggest that appropriate management for osteoporosis in patients with RA is crucial not only for postmenopausal women, but also for men aged 50 years and over.

Disclosure of Interest: None declared

DOI: 10.1136/annrheumdis-2017-eular.3061

\section{FRI0551 USE OF GLUCOCORTICOID IS THE RISK FACTOR FOR INADEQUATE RESPONSE TO THE TREATMENT OF OSTEOPOROSIS BY DENOSUMAB}

K. Orita ${ }^{1}$, T. Koike ${ }^{2} .{ }^{1}$ Orthopaedic Surgery, Shirahama Hamayu Hospital, Shirahama Foundation for Health and Welfare; ${ }^{2}$ Orthopaedic Surgery, 2. Search Institute for Bone and Arthritis Disease (SINBAD), Shirahama Foundation for Health and Welfare, Shirahama, Japan

Background: In treating rheumatoid arthritis (RA), T2T (treat-to-Target) is the most reliable treatment strategy. Recent reports have indicated that reaching normal levels of bone mineral density (BMD) might be important for the prevention of fractures in osteoporosis treatment (reference 1 and 2).From this fact, there might be a possibility that T2T targeting BMD might be feasible also in osteoporosis treatment. In doing so, medicines with the ability to sufficiently increase BMD at a fast speed should be needed. Denosumab (DMAb) specifically inhibits the receptor activator for nuclear factor-kappa $B$ ligand (RANKL) improves BMD rapidly at lumbar or hip. Therefore, DMAb is one of candidate drugs for T2T 
practice, and it is very important to know risk factors that attenuate its effect in the treatment of osteoporosis.

Objectives: The aim of this study was to identify risk factors for inadequate response to the treatment of osteoporosis by DMAb.

Methods: Sixty-six patients treated with DMAb were observed retrospectively for one year. The mean age was 74 years, women were $91 \%$ and 36 patients with RA were included.We measured BMDs at lumbar and hip by dual-energy X-ray absorptiometry (Hologic Discovery) at baseline and one year later. We evaluated the effects of age, body mass index (BMI), use of glucocorticoide (GC), previous treatment for osteoporosis, BMD at baseline, bone metabolic markers (BAP; bone alkaline phosphatase, uNTx; urinary N-telopeptide), serum $\mathrm{Ca}$ and $\mathrm{P}$ levels and the previous vertebral fractures for inadequate response to DMAb. We defined the cases who could not gain the increase of BMD over $2 \%$ at the lumbar vertebrae and $4 \%$ at the hip as inadequate responders by taking the measurement error into account.

Results: Dose of PSL was significantly high in non-responder at non-RA trochanter and RA lumbar BMD $(p=0.028,0.006)$. BAP was higher in nonresponder at RA lumbar BMD $(p=0.007)$. Urinary NTX was significantly low in non-responder at non-RA lumbar and RA trochanter BMD $(p=0.026,0.048)$. Previous treatment for osteoporosis was significantly high in non-responder at non-RA lumbar, total hip and trochanter BMD $(\mathrm{p}=0.026,0.022,0.003)$. Multivariate logistic analysis including age, BMI, dose of PSL, BMD at baseline, BAP, UNTX, $\mathrm{Ca}$ and $\mathrm{P}$ level as confounders revealed that dose of PSL was the significant risk factors for no-response at lumbar BMD (OR0.634, 95\% Cl 0.433-0.93, $\mathrm{p}=0.02$ ). Conclusions: Patients receiving GC might not gain an adequate response to the treatment by DMAb for osteoporosis. Reducing dose of GC or altanative treatment regimen might be necessary.

References:

[1] Kuroda T. et al. J Clin Densitom 15: 392-8, 2012.

[2] Bauer D.C. et al. JAMA 174: 1126-34, 2014.

Disclosure of Interest: None declared

DOI: 10.1136/annrheumdis-2017-eular.3684

\section{FRI0552 THE CORRELATION BETWEEN EROSION AND BONE MINERAL DENSITY IN PATIENTS WITH RHEUMATOID ARTHRITIS TREATED WITH DENOSUMAB AND BIOLOGIC DMARDS: A PROSPECTIVE COHORT STUDY}

K. Izumi, Y. Kaneko, T. Hasegawa, T. Takeuchi. Division of Rheumatology, Department of Internal Medicine, Keio University School of Medicine, Tokyo, Japan

Background: We have previously reported the efficacy of denosumab combined with biological DMARDs on radiographic progression in rheumatoid arthritis (RA). Objectives: The aim of this work was to reveal the relationship between the changes in structural joint damage and in bone mineral density (BMD) in patients with RA who were started on denosumab in addition to biologic DMARDs.

Methods: We prospectively evaluated erosion (ERO) and joint space narrowing (JSN) scores by the van der Heijde-modified Sharp method and T-scores of the lumbar spine (LS) and total hip (TH) by dual energy X-ray absorptiometry scans at baseline and 12 months in the RA patients who were started on denosumab (60 mg every 6 months) for osteoporosis in addition to biologic DMARDs. We compared the 12-month change in ERO or JSN scores ( $\triangle E R O ; \triangle J S N)$ with the change in T-scores of the LS or TH $(\Delta \mathrm{LS} ; \Delta \mathrm{TH})$.

Results: Twenty-two patients ( 1 man and 21 women) at the mean \pm SD age of $74.4 \pm 8.1$ at baseline were included in this study. The T-scores of the LS and TH at baseline were $-1.38 \pm 1.57$ and $-2.53 \pm 0.85$, respectively. $\triangle \mathrm{ERO}, \Delta \mathrm{JSN}, \Delta \mathrm{LS}$, and $\Delta \mathrm{TH}$ were $0.16 \pm 0.49,0.44 \pm 0.66,0.30 \pm 0.39$, and $0.20 \pm 0.42$, respectively. BMD was significantly increased from baseline to 12 months. There was a significant inverse correlation between the $\Delta E R O$ and the $\Delta T H(\rho=-0.473, P=0.03)$, while there was no correlation between the $\triangle E R O$ and the $\Delta L S(P=0.98)$ nor correlation between the $\triangle J S N$ and the $\Delta L S(P=0.57)$ or $\Delta T H(P=0.25)$.

Conclusions: The change in erosive joint damage of hands and feet showed a significant relationship with the change in femoral BMD in patients with RA treated with denosumab and biologic DMARDs. BMD was ameliorated along with erosion by denosumab combined with biologic DMARDs, and may be an indicator for joint destruction.

References:

[1] Hasegawa T, Kaneko Y, Izumi K, Takeuchi T. Efficacy of denosumab combined withbDMARDs on radiographic progression in rheumatoid arthritis. Joint Bone Spine.2016 Jun 28. pii: S1297-319X(16)30099-9.

Disclosure of Interest: None declared

DOI: 10.1136/annrheumdis-2017-eular.2829

\section{FRI0553 THE EFFICACY OF 2-YEARS DENOSUMAB TREATMENT FOR GLUCOCORTICOID-INDUCED OSTEOPOROSIS (GIOP)}

K. Akashi $^{1}$, K. Nishimura ${ }^{2}$, G. Kageyama ${ }^{3}$, S. Ichikawa ${ }^{1}$, T. Shirai ${ }^{1}$, Y. Yamamoto ${ }^{1}$, Y. Ichise ${ }^{1}$, H. Yamada ${ }^{1}$, I. Naka ${ }^{1}$, D. Waki ${ }^{1}$, T. Okano ${ }^{1}$ S. Takahashi ${ }^{1}$, Y. Ueda ${ }^{1}$, S. Sendo ${ }^{1}$, A. Onishi ${ }^{1}$, J. Saegusa ${ }^{1}$, A. Morinobu ${ }^{1}$. ${ }^{1}$ Department of Rheumatology and Clinical Immunology, Kobe University Hospital, Kobe, ${ }^{2}$ Department of Endocrinology and Rheumatology, Kurashiki
Central Hospital, Kurashiki; ${ }^{3}$ Department of Rheumatology, Hyogo Prefectual Amagasaki General Medical Center, Amagasaki, Japan

Background: Osteoporosis is one of the important adverse effects in the glucocorticoids treatment for the patients with rheumatoid arthritis (RA) and connective tissue diseases (CTDs). Although the usefulness of denosmab for primary osteoporosis has been well-established, the efficacy for GIOP remains unclear.

Objectives: This study aimed to clarify the therapeutic effects of denosumab for GIOP.

Methods: We evaluated bone mineral density (BMD) and serum markers of bone metabolism (BAP, NTx, TRACP-5b and P1NP) of patients who had been treated with over $5 \mathrm{mg}$ of predonisolone for RA and CTDs, and denosumab for GIOP, for two years in Kobe University Hospital. BMD and serum markers were evaluated every six months for 2 years from the baseline. The changes of those data from baseline were analyzed by Student's t test using GraphPad Prism 5 software and $\mathrm{p}<0.05$ was considered statistically significant.

Results: Number of the patients were 53 (male: 4 cases, female: 49 cases), and their characteristics at the beginning of denosumab treatment were as below; age: $64.19 \pm 12.0$ years old, dose of prednisolon: $10.59 \pm 9.97 \mathrm{mg} /$ day, BMD of lumber spine: $0.768 \pm 0.112 \mathrm{~g} / \mathrm{cm}^{3}$, T-score of lumber spine: $-2.28 \pm 1.01$, BMD of femoral neck: $0.540 \pm 0.085 \mathrm{~g} / \mathrm{cm}^{3}$, T-score of femoral neck: $-2.28 \pm 0.76$. After 2 -years denosumab treatment, T-scores of lumber spine $(0.54 \pm 0.39$ gain $)$ and femoral neck $(0.13 \pm 0.26$ gain) were significantly increased from baseline (Figure; mean \pm SEM. $\left.{ }^{*}: p<0.05\right)$. In addition, the serum markers of bone metabolism, both absorption and formation, were significantly suppressed with denosumab.
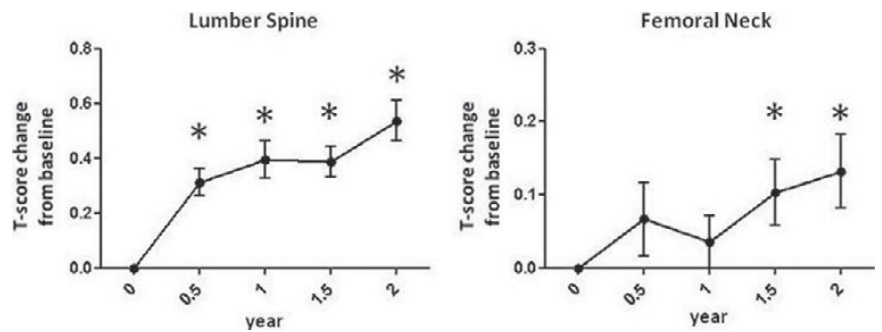

Conclusions: Denosumab can suppress bone metabolic turnover, and increase lumber spine and femoral neck T-scores of GIOP patients.

Disclosure of Interest: None declared

DOI: 10.1136/annrheumdis-2017-eular.3050

\section{FRI0554 NEW BIOMARKER FOR DIAGNOSIS OF OSTEOPOROSIS IN RHEUMATOID ARTHRITIS PATIENTS}

Y. Polyakova, L. Sivordova, Y. Akhverdyan, V. Kravtcov, B. Zavodovsky. Federal State Budgetary Institution "Research Institute of Clinical end Experimental Rheumatology", Volgograd, Russian Federation

Background: Bone mineral density and proteins/peptides determination in blood and urine as markers of bone resorption and formation are currently used to diagnose osteoporosis (OP) and metabolic bone diseases. However, these methods have some disadvantages for bone turnover evaluation. Recent evidence suggests that in RA changes in the secretion of hormones of white adipose tissue can be revealed [2]. One of them is Adiponectin possessing anti-inflammatory, anti-diabetic and anti-atherogenic properties [1]. Changes in Adiponectin levels may reflect influence of immune inflammation on bone turnover.

Objectives: To study the clinical and diagnostic value of serum Adiponectin determination in RA patients complicated by OP.

Methods: We examined 88 women with documented diagnosis of RA and mean disease duration of $6.56 \pm 0.88$ years. We used EULAR/ARA 2010 criteria to diagnose the patients. Female patients with II degree of disease activity (DAS28), Steinbrocker stage II (erosive), rheumatoid factor- and anti-cyclic-citrullinated peptide antibody-positive were prevalent. We excluded patients who had surgery or developed an infection within the last 8 weeks, pregnant and breast-feeding women, those with severe heart, liver or kidney disease, immune deficiency, leukopenia or chronic infection.

A control group of 45 healthy females aged of 25 and 59 years were included in the study. There were no reported findings of joint pain and RA symptoms in the group. The groups were adjusted for age $(p>0.05)$ and showed no statistically significant differences.

We measured serum Adiponectin levels $(\mu \mathrm{g} / \mathrm{ml})$ using Human Adiponectin ELISA commercial test systems. We plotted a curve using computer software. We diagnosed OP using dual-energy X-ray absorptiometry with LUNAR DPX PRO (GE, USA)

Results: Serum adiponectin levels in the control group were $12.5 \pm 0.9 \mu \mathrm{g} / \mathrm{ml}$ $(\mathrm{M} \pm \mathrm{m})$. Adiponectin levels in healthy subjects measured as $\mathrm{M} \pm 2 \mathrm{~d}$, ranged between 0.44 and $24.56 \mu \mathrm{g} / \mathrm{ml}$. Patients with OP and RA had significantly higher levels of serum Adiponectin $(p<0.001)$. Mean serum Adiponectin levels in RA patients who had normal bone density and had no OP were $35.21 \pm 0.6$ $\mu \mathrm{g} / \mathrm{ml}$. Mean serum Adiponectin levels in RA/OP patients with low bone mineral density were $52.42 \pm 0.69 \mu \mathrm{g} / \mathrm{ml}$. Adiponectin levels of $44 \mu \mathrm{g} / \mathrm{ml}$ and higher were 